\title{
A!
}

This is an electronic reprint of the original article.

This reprint may differ from the original in pagination and typographic detail.

Ramires, Aline; Lado, Jose

\section{Emulating Heavy Fermions in Twisted Trilayer Graphene}

Published in:

Physical Review Letters

DOI:

10.1103/PhysRevLett.127.026401

Published: 09/07/2021

Document Version

Publisher's PDF, also known as Version of record

Please cite the original version:

Ramires, A., \& Lado, J. (2021). Emulating Heavy Fermions in Twisted Trilayer Graphene. Physical Review Letters, 127(2), 1-6. [026401]. https://doi.org/10.1103/PhysRevLett.127.026401

This material is protected by copyright and other intellectual property rights, and duplication or sale of all or part of any of the repository collections is not permitted, except that material may be duplicated by you for your research use or educational purposes in electronic or print form. You must obtain permission for any other use. Electronic or print copies may not be offered, whether for sale or otherwise to anyone who is not an authorised user. 


\title{
Emulating Heavy Fermions in Twisted Trilayer Graphene
}

\author{
Aline Ramires $\circledast^{1, *}$ and Jose L. Lado $\odot^{2, \dagger}$ \\ ${ }^{1}$ Condensed Matter Theory Group, Paul Scherrer Institute, CH-5232 Villigen PSI, Switzerland \\ ${ }^{2}$ Department of Applied Physics, Aalto University, 00076 Aalto, Espoo, Finland
}

(Received 21 February 2021; accepted 8 June 2021; published 7 July 2021)

\begin{abstract}
Twisted van der Waals materials have been shown to host a variety of tunable electronic structures. Here we put forward twisted trilayer graphene (TTG) as a platform to emulate heavy fermion physics. We demonstrate that TTG hosts extended and localized modes with an electronic structure that can be controlled by interlayer bias. In the presence of interactions, the existence of localized modes leads to the development of local moments, which are Kondo coupled to coexisting extended states. By electrically controlling the effective exchange between local moments, the system can be driven from a magnetic into a heavy fermion regime, passing through a quantum critical point, allowing one to electrically explore a generalized Doniach phase diagram. Our results put forward twisted graphene multilayers as a platform for the realization of strongly correlated heavy fermion physics in a purely carbon-based platform.
\end{abstract}

DOI: 10.1103/PhysRevLett.127.026401

Twisted van der Waals multilayers have recently risen as a new family of systems allowing for the realization of multiple exotic quantum states of matter [1-10]. Their versatility stems from the control of the strength of electronic correlations and emergent topological properties by means of the twist angle between the different layers [11-19]. As paradigmatic examples, twisted graphene bilayers have realized unconventional superconductivity [3], topological networks [6], strange metals [20], and Chern insulators [9]. More complex twisted graphene multilayers, such as twisted bi-bilayers $[7,10]$ and trilayers [21-23], have provided additional platforms to realize similar physics as twisted graphene bilayers. The possibility of tuning several twist angles in multilayer graphene [24-28] suggests that these systems may realize correlated states of matter beyond the ones already observed in twisted bilayers.

Heavy fermions are a family of strongly correlated materials hosting a variety of fascinating quantum manybody states $[29,30]$ and unusual quantum critical phenomena [31-34]. Two main ingredients constitute the electronic degrees of freedom in heavy fermions: localized states, usually associated with $f$ orbitals, and a sea of delocalized electrons. In these materials, strong interactions in the localized degrees of freedom generate local magnetic moments, which, through the Kondo effect, couple to the surrounding conduction electrons and dramatically renormalize their mass. Remarkably, graphene multilayers are known to host both dispersive [6,35] and localized electronic states $[2,11,36]$. However, the coexistence of dispersive and localized modes does not occur in the most studied instances of twisted graphene bilayers and bi-bilayers. (a)

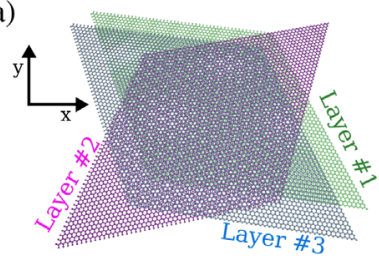

(c)

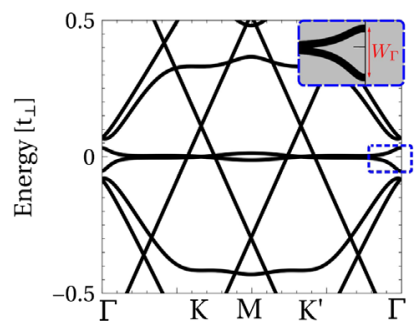

(e)

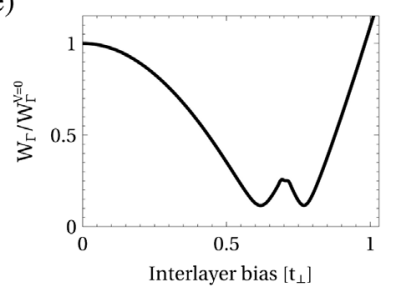

(b)
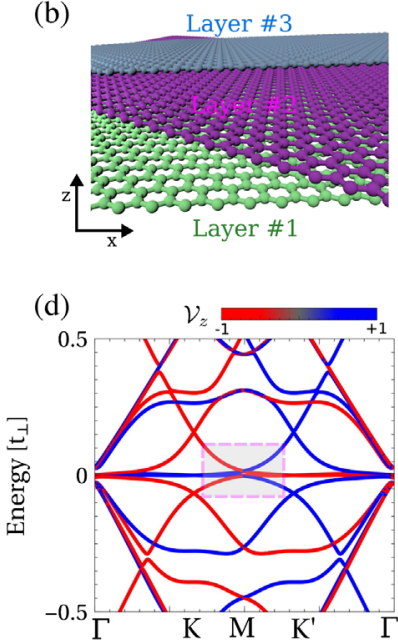

(f)

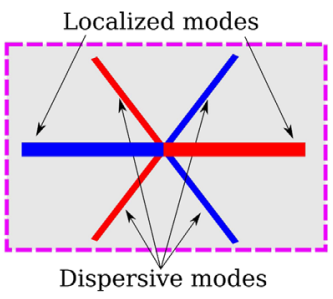

FIG. 1. Top (a) and side (b) view of the twisted trilayer graphene system. (c),(d) Electronic structure in the absence and presence of interlayer bias $V=0.6 t_{\perp}$, respectively. Color code in (d) corresponds to the valley quantum number $\mathcal{V}_{z}$. (e) Dependence of the flat band width, defined in the inset of (c), on the interlayer bias. (f) Schematic represents the low energy sector with localized and itinerant modes. 
Here we establish that twisted graphene trilayers can host electronic states that mimic heavy fermion systems in a purely carbon-based material. We show that the main ingredients, flat bands, and highly dispersive states appear simultaneously and provide the starting point to engineer and control electronic phases analogous to the ones observed in heavy fermions. We discuss how, in the presence of interactions, the highly degenerate flat bands lead to the formation of local spin and valley moments, which become exchange coupled to dispersive helical modes, leading to the emergence of heavy fermions. Our proposal allows one to electrically explore a Doniach phase diagram, putting forward a tunable platform for heavyfermion physics in van der Waals materials.

We start by introducing the atomistic Hamiltonian for the twisted trilayer, demonstrating that these systems host the fundamental ingredients to realize heavy fermion physics. We take a single orbital per carbon atom, yielding a tightbinding Hamiltonian of the form $[11,15]$

$$
\begin{aligned}
\mathcal{H}= & -t \sum_{\langle i, j\rangle, s} c_{i, s}^{\dagger} c_{j, s}-\sum_{i, j, s} \bar{t}_{\perp}\left(\mathbf{r}_{i}, \mathbf{r}_{j}\right) c_{i, s}^{\dagger} c_{j, s} \\
& +\frac{V}{2 d} \sum_{i, s} z_{i} c_{i, s}^{\dagger} c_{i, s},
\end{aligned}
$$

where $c_{i, s}^{\dagger}\left(c_{i, s}\right)$ creates (annihilates) electrons at site $i$ with spin $s$. In the first term, $t$ is the intralayer hopping amplitude, and $\langle i, j\rangle$ restricts the sum to first neighbors. The second term accounts for interlayer hopping defined as $\bar{t}_{\perp}\left(\mathbf{r}_{i}, \mathbf{r}_{j}\right)=t_{\perp}\left\{\left[\left(z_{i}-z_{j}\right)^{2}\right] /\left[\left|\mathbf{r}_{i}-\mathbf{r}_{j}\right|^{2}\right]\right\} e^{-\beta\left(\left|\mathbf{r}_{i}-\mathbf{r}_{j}\right|-d\right)} \quad[11,15]$, where $d$ is the interlayer distance and $\beta$ controls the spatial decay of the hopping amplitudes. As a reference, for twisted graphene multilayers $t \approx 3 \mathrm{eV}$ and $t_{\perp} \approx 0.15 t$ [37]. The third term corresponds to interlayer bias with magnitude $V$, with $z_{i}= \pm d$ for the upper/lower layer and $z_{i}=0$ for the middle layer. Here we consider a twisted trilayer structure with the top and bottom layers aligned, rotated by an angle $\theta$ with respect to the middle layer, as shown in Figs. 1(a) and 1(b).

The electronic structure for $\theta=1.6^{\circ}$ is shown in Fig. 1(c) for $V=0$. We highlight the emergence of flat bands, similar to the ones observed in twisted bilayer graphene at the magic angle, coexisting with dispersive modes [25-28,38]. Interestingly, when applying an interlayer bias, this twisted system develops even flatter bands, as shown in Fig. 1(d), in stark contrast with the case of magic-angle bilayers, for which an interlayer bias does not impact the electronic structure strongly [5]. This can be quantitatively assessed by computing the splitting of the nearly flat bands at the $\Gamma$ point as a function of the interlayer bias, as shown in Fig. 1(e). In particular, it is observed that for interlayer biases of $V \approx 0.5 t_{\perp}$ the bandwidth becomes drastically reduced. Moreover, in the presence of interlayer bias, the electronic states can still be associated with a well-defined valley quantum number
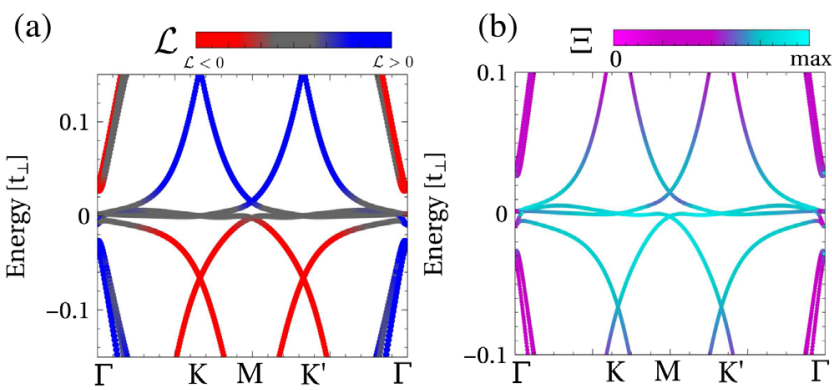

(c)

(d)

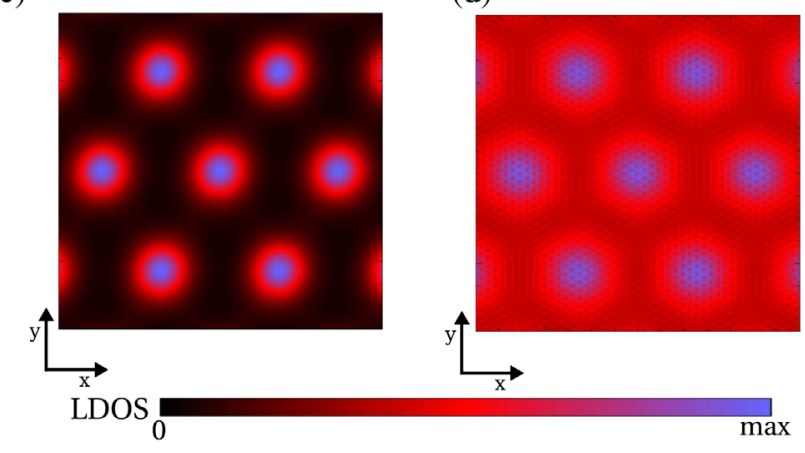

FIG. 2. (a),(b) Band structure of the biased $\left(V=0.6 t_{\perp}\right)$ twisted trilayer, highlighting the layer polarization of the states, $\mathcal{L}$, in (a), and their localization in the unit cell, quantified by the inverse participation ratio $\Xi$, in (b). (c),(d) Local density of states for the flat modes at $\omega=0$ (c) and for the dispersive modes at $\omega=$ $0.03 t_{\perp}(\mathrm{d})$.

$\mathcal{V}_{z}$ (computed in the real-space basis using the valley operator [39-43]). In summary, we have the coexistence of valley polarized and spin degenerate ultraflat states and dispersive modes, as shown schematically in Fig. 1(f).

We now focus on the flattest regime in the presence of a bias, as shown in Fig. 2. By computing the layer polarization, $\mathcal{L}$, shown in Fig. 2(a), we conclude that both the flat bands and dispersive modes are delocalized between the three layers, with dispersive modes showing a small layer polarization. Moreover, the localization of the states in the moiré unit cell can be characterized by means of the inverse participation ratio $\Xi=\sum_{i}\left|\Psi_{n}(i)\right|^{4}$, Fig. 2(b). It is observed that the flat bands are strongly localized in the moiré unit cell, whereas the dispersive modes become more delocalized. The different localization can be directly observed from the local density of states (LDOS) at the energy of the localized and dispersive modes, shown in Figs. 2(c) and 2(d), respectively.

To understand how the electronic structure is modified in the presence of interactions, we first study the problem at the mean-field level. This is equivalent to first-principles electronic structure calculations performed for heavy fermion compounds [44-50]. Electronic interactions for the atomistic Hamiltonian take the form $\mathcal{H}_{\text {int }}=$ $\sum_{i j} v\left(\mathbf{r}_{i}, \mathbf{r}_{j}\right) \rho_{i} \rho_{j}$, where $\rho_{i}=\sum_{s} c_{i, s}^{\dagger} c_{i, s}$, and $v\left(\mathbf{r}_{i}, \mathbf{r}_{j}\right)$ is a screened Coulomb interaction. A mean-field decoupling allows one to transform the previous density-density 
(a)

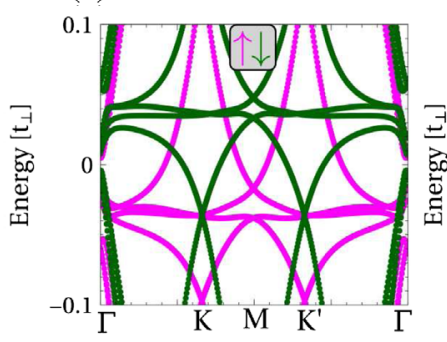

(c)

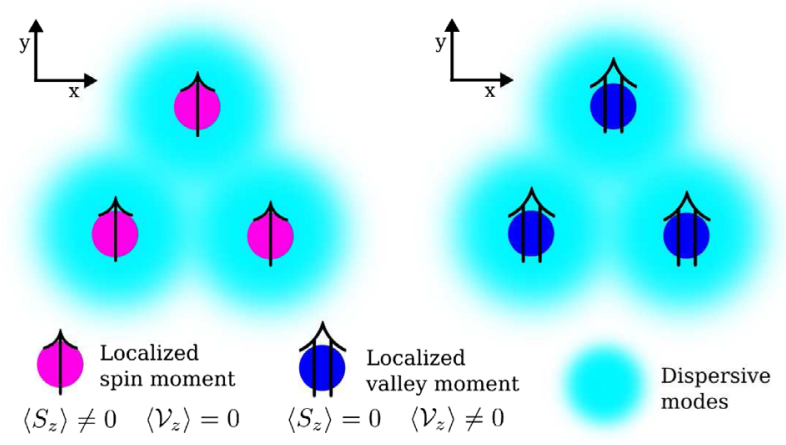

FIG. 3. (a) Band structure in the presence of finite spin (a) and valley (b) polarization of the localized modes. (c),(d) Sketch of the order associated with the bandstructures in (a) and (b), respectively.

operator into a single-particle operator of the form $H^{M F}=\sum_{i j s s^{\prime}} \chi_{i j}^{s s^{\prime}} c_{i, s}^{\dagger} c_{j, s^{\prime}}$, where $\chi_{i j}^{s s^{\prime}}$ are the mean-field field parameters. In the following, we will consider two mean-field ansatzes, one leading to a mean field with spin polarization in the localized modes, and one leading to valley polarization in the localized modes. It must be noted that, although such mean-field electronic structure does not capture quantum fluctuations, it can be taken as an estimate of the backaction of the localized modes onto the dispersive states. As a reference, in our calculations, we take an electronic repulsion equivalent to a charging energy of $50 \mathrm{meV}$, comparable to the one of twisted bilayer graphene [51-55]. It is finally worth noting that the strength of the Coulomb interactions can be externally controlled by means of screening engineering through a substrate [52,54].

The mean-field band structures of the atomistic model for the case of a finite spin and valley polarization are shown in Figs. 3(a) and 3(b), respectively, highlighting that the polarization of the localized modes leads to strong and qualitatively distinct band reconstructions. This phenomenology is analogous to the one found in twisted bilayers $[51,53,55]$, with the key difference that twisted bilayers do not have dispersive modes coexisting at the Fermi energy. In the proposed twisted trilayer graphene system, these results highlight that the dispersive modes are strongly affected by the localized ones, signaling the existence of exchange coupling between extended and localized modes, what can be understood in terms of a Kondo coupling [56-59].
The phenomenology obtained from the microscopic atomistic model within a mean-field approach shows that biased twisted trilayers have the fundamental ingredients to display heavy fermion physics. In order to proceed with the discussion, we now focus on the low energy effective model for a twisted trilayer graphene, captured by

$$
H=H_{\text {Helical }}+H_{\mathrm{LM}}+H_{\mathrm{Hyb}}+H_{\mathrm{Int}} .
$$

Here $H_{\text {Helical }}$ corresponds to the propagating helical dispersive modes. Note that, in contrast to the usual concept of helicity locking the direction of propagation with the spin projection, here the helical modes have the direction of propagation locked with the valley DOF, while they are spin degenerate:

$$
H_{\text {Helical }} \approx v_{F} \sum_{k>0, \sigma} k d_{k \Uparrow \sigma}^{\dagger} d_{k \Uparrow \sigma}+v_{F} \sum_{k<0, \sigma} k d_{k \Downarrow \sigma}^{\dagger} d_{k \Downarrow \sigma},
$$

where $\sigma=\{\uparrow, \downarrow\}$ and $v=\{\Uparrow, \downarrow\}$ correspond to the spin and valley degrees of freedom (DOF), respectively, $k$ denotes the wave vector difference with respect to the Fermi surface, and $v_{F}$ stands for the Fermi velocity. The flat bands are accounted as localized states at each moiré site with internal spin and valley DOFs:

$$
H_{\mathrm{LM}}=\sum_{i, \sigma, v} \epsilon_{\mathrm{LM}} f_{i v \sigma}^{\dagger} f_{i v \sigma} .
$$

From the atomistic calculations, Fig. 1(b), we access that the hybridization between the localized and helical modes preserves spin and valley DOF; therefore, we write

$$
H_{\mathrm{Hyb}}=\delta \sum_{i, \sigma, v} f_{i v \sigma}^{\dagger} d_{i v \sigma}+\text { H.c. }
$$

where $\delta$ is the hybridization strength. As a reference, our calculations show an effective hybridization strength of $\delta \approx 5 \mathrm{meV}$. The interaction Hamiltonian penalizes multiple occupancy of the flat band modes

$$
H_{\mathrm{Int}}=U \sum_{i, v, v^{\prime}, \sigma, \sigma^{\prime}} n_{i f, v \sigma} n_{i f, v^{\prime} \sigma^{\prime}}
$$

where $U$ is a generalized Hubbard interaction parameter. The sum should exclude terms with both $v=v^{\prime}$ and $\sigma=\sigma^{\prime}$. Interactions in the dispersive modes would lead to a renormalization of the Fermi velocity, and are reabsorbed in $H_{\text {Helical }}$ [60-65]. We note that it is possible to write a more generic interorbital coupling, yet SU(4) models are known to effectively account for the interactions of localized modes in graphene multilayers [66,67].

We can now find an effective Kondo model by integrating out the high energy DOF of the generalized Anderson model. This can be done through a Schrieffer-Wolf transformation. In particular, from our atomistic calculations 
we obtain $U=50 \mathrm{meV}$ and $\delta \approx 5 \mathrm{meV}$, which fulfill the $\delta \ll U$ criteria required to map the Anderson model to the Kondo model. Note that if the assumption of a spin and valley conserving hybridization holds, we can assign a unique index $\alpha=(v, \sigma)$ summed over four flavors, such that the problem can be casted as a $\mathrm{SU}(4)$ Kondo model [68-73]:

$$
H_{\text {Kondo }}=H_{\text {Helical }}+J_{K} \sum_{i} \mathbf{S}_{i} \cdot \mathbf{s}_{i},
$$

where $J_{K} \approx \delta^{2} / U$ is the Kondo coupling, $\mathbf{S}_{i}=\Psi_{f i}^{\dagger} \boldsymbol{\Gamma} \Psi_{f i}$, and $\mathbf{s}_{i}=\Psi_{d i}^{\dagger} \boldsymbol{\gamma} \Psi_{d i}$. Here $\boldsymbol{\Gamma}$ and $\boldsymbol{\gamma}$ stand for the array of 15 generators of $\mathrm{SU}(4)$ plus the identity, and $\Psi_{f i}^{\dagger}=$ $\left(f_{i \Uparrow \uparrow}^{\dagger}, f_{i \Uparrow \downarrow}^{\dagger}, f_{i \Downarrow \uparrow}^{\dagger}, f_{i \Downarrow \downarrow}^{\dagger}\right)$ and $\Psi_{d i}^{\dagger}=\left(d_{i \Uparrow \uparrow}^{\dagger}, d_{i \Uparrow \downarrow}^{\dagger}, d_{i \Downarrow \uparrow}^{\dagger}, d_{i \Downarrow \downarrow}^{\dagger}\right)$. Interestingly, the effect of enlarging the symmetry group associated with the degrees of freedom that can scatter through the Kondo coupling by generalizing the Kondo effect from $\mathrm{SU}(2)$ to $\mathrm{SU}(\mathrm{N})$ enhances the Kondo temperature according to $T_{K}^{S U(N)} \approx D\left(N \rho_{0} J_{K}\right)^{1 / N} e^{1 /\left(N \rho_{0} J_{K}\right)}$, where $\rho_{0}$ is the flat density of states and $D$ the bandwidth associated with the helical modes [71,72]. Note that the Kondo temperature is exponentially enhanced by increasing $N$ [74-78]. The fact that the delocalized states are helical does not play a critical role in the Kondo effect and on the renormalization procedure as long as the DOS is finite $[79,80]$.

The existence of the heavy-fermion regime requires that Kondo interaction $J_{K}$ dominates the exchange coupling between localized moments $J$. As a reference, our atomistic calculations yield an effective Kondo coupling of the order of $J_{K} \approx 0.5 \mathrm{meV}$. In the $\mathrm{SU}(4)$ scenario, the exchange coupling between local modes takes the form $H_{\text {Exc }}=J \sum_{\langle i j\rangle} \mathbf{S}_{i} \cdot \mathbf{S}_{j}$. Interestingly, exchange couplings in twisted van der Waals materials have been shown to be tunable all the way from ferromagnetic to antiferromagnetic $[16,43,81]$. We now compute the exchange $J$ between two neighboring localized modes by means of the magnetic force theorem [56-59]. In the absence of interlayer bias, we obtain a ferromagnetic coupling between local moments of $|J(V=0)| \approx 2 \mathrm{meV}$ [82]. Once an interlayer bias is applied, the exchange coupling is reduced and can even change sign for increasing bias, as shown in Fig. 4(b). In particular, we obtain that the exchange between local moments can be switched off for $V \approx 0.5 t_{\perp}$. In this regime, the Kondo coupling $J_{K}$ is the dominant interaction, driving the twisted trilayer to the heavy-fermion regime. Importantly, depending on the sign of $J$, the phase diagram of the twisted trilayer will take two different forms. For ferromagnetic coupling $J<0$, a quantum critical point separates the magnetically ordered phase from the Kondo screened phase, Fig. 4(c). In comparison, for antiferromagnetic coupling $J>0$, the geometric frustration of the superlattice potentially leads to a quantum critical
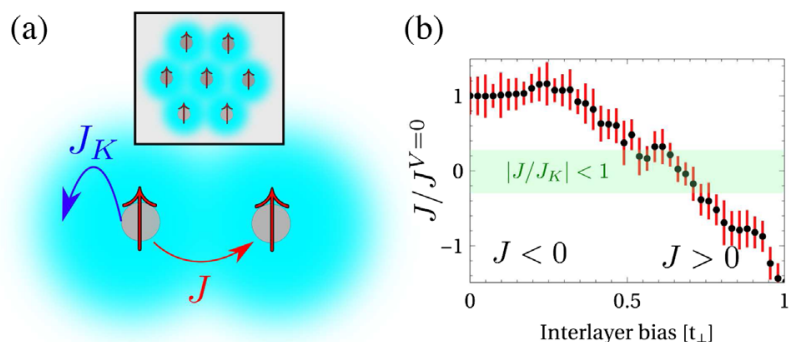

(c)

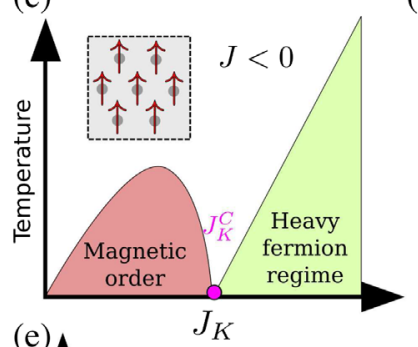

(d)

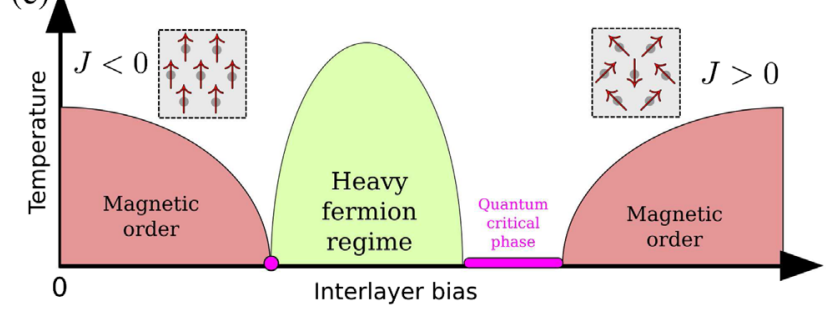

FIG. 4. (a) Sketch of the exchange between local moments of strength $J$, and the Kondo coupling between extended and localized modes of strength $J_{K}$. (b) Dependence of the exchange coupling with the interlayer bias, showing an electrical switch from ferromagnetic to antiferromagnetic. Green region in (b) shows the regime in which $\left|J / J_{K}\right|<1$. (c) Phase diagram in the presence of ferromagnetic exchange and (d) in the presence of antiferromagnetic exchange. (e) Image of how the three phases can be explored by applying an interlayer bias between the layers, stemming from the exchange control shown in (b).

phase for a finite range of couplings [32,83-85], as shown in Fig. 4(d).

Interestingly, the electric tunability of the twisted trilayer allows for the exploration of these two types of phase diagram with a single control parameter, the interlayer bias, as displayed in Fig. 4(e). This finding proposes a new perspective on the global phase diagram for heavy fermions $[33,34]$. While a precise first-principles estimate of all the couplings is challenging [25], the exact system's parameters can be inferred from experiments looking at the evolution of the tunneling spectra as a function of temperature and magnetic fields in different directions. In particular, below the coherence temperature, Fano-like resonances appear and the characteristic parameters of the line shape can be extracted and identified with model parameters such as the hybridization between localized and delocalized states [86-90].

To summarize, we have shown, combining accurate atomistic calculations and low energy effective models, 
that twisted graphene trilayers provide a van der Waals platform to engineer heavy fermion physics. In particular, we demonstrated that the existence of electrically controllable flat bands and dispersive modes provides the singleparticle starting point to simulate Kondo lattices. In the presence of interactions, the flat bands lead to local spin and valley moments. Interestingly, the electric tunability of the intermoment exchange couplings allows for the exploration of both the conventional and frustrated Kondo lattice regimes, such that twisted trilayers can realize the global Doniach phase diagram for heavy fermions within a single material platform. Our results show that twisted graphene multilayers provide a carbon-only platform to emulate rare earth compounds, opening new possibilities in the field of correlated twisted van der Waals materials.

A. R. acknowledges financial support from SNSF Ambizione. J.L. L. acknowledges the computational resources provided by the Aalto Science-IT project, the financial support from the Academy of Finland Projects No. 331342 and No. 336243, and from the Jane and Aatos Erkko Foundation. We thank M. Sigrist, T. Neupert, P. Liljeroth, and P. Rickhaus for useful discussions.

*aline.ramires@psi.ch

jose.1ado@aalto.fi

[1] G. Li, A. Luican, J. M. B. L. dos Santos, A. H. C. Neto, A. Reina, J. Kong, and E. Y. Andrei, Nat. Phys. 6, 109 (2010).

[2] Y. Cao, V. Fatemi, A. Demir, S. Fang, S. L. Tomarken, J. Y. Luo, J. D. Sanchez-Yamagishi, K. Watanabe, T. Taniguchi, E. Kaxiras, R. C. Ashoori, and P. Jarillo-Herrero, Nature (London) 556, 80 (2018).

[3] Y. Cao, V. Fatemi, S. Fang, K. Watanabe, T. Taniguchi, E. Kaxiras, and P. Jarillo-Herrero, Nature (London) 556, 43 (2018).

[4] X. Lu, P. Stepanov, W. Yang, M. Xie, M. A. Aamir, I. Das, C. Urgell, K. Watanabe, T. Taniguchi, G. Zhang, A. Bachtold, A. H. MacDonald, and D. K. Efetov, Nature (London) 574, 653 (2019).

[5] M. Yankowitz, S. Chen, H. Polshyn, Y. Zhang, K. Watanabe, T. Taniguchi, D. Graf, A. F. Young, and C. R. Dean, Science 363, 1059 (2019).

[6] P. Rickhaus, J. Wallbank, S. Slizovskiy, R. Pisoni, H. Overweg, Y. Lee, M. Eich, M.-H. Liu, K. Watanabe, T. Taniguchi, T. Ihn, and K. Ensslin, Nano Lett. 18, 6725 (2018).

[7] Y. Cao, D. Rodan-Legrain, O. Rubies-Bigorda, J. M. Park, K. Watanabe, T. Taniguchi, and P. Jarillo-Herrero, Nature (London) 583, 215 (2020).

[8] P. Rickhaus, F. de Vries, J. Zhu, E. Portolés, G. Zheng, M. Masseroni, A. Kurzmann, T. Taniguchi, K. Wantanabe, A. H. MacDonald, T. Ihn, and K. Ensslin, arXiv:2005.05373.

[9] M. Serlin, C. L. Tschirhart, H. Polshyn, Y. Zhang, J. Zhu, K. Watanabe, T. Taniguchi, L. Balents, and A.F. Young, Science 367, 900 (2020).
[10] X. Liu, Z. Hao, E. Khalaf, J. Y. Lee, Y. Ronen, H. Yoo, D. H. Najafabadi, K. Watanabe, T. Taniguchi, A. Vishwanath, and P. Kim, Nature (London) 583, 221 (2020).

[11] E. Suárez Morell, J. D. Correa, P. Vargas, M. Pacheco, and Z. Barticevic, Phys. Rev. B 82, 121407(R) (2010).

[12] E. Suárez Morell, P. Vargas, L. Chico, and L. Brey, Phys. Rev. B 84, 195421 (2011).

[13] J. M. B. Lopes dos Santos, N. M. R. Peres, and A. H. Castro Neto, Phys. Rev. Lett. 99, 256802 (2007).

[14] R. Bistritzer and A. H. MacDonald, Proc. Natl. Acad. Sci. U.S.A. 108, 12233 (2011).

[15] A. O. Sboychakov, A. L. Rakhmanov, A. V. Rozhkov, and F. Nori, Phys. Rev. B 92, 075402 (2015).

[16] L. A. Gonzalez-Arraga, J. L. Lado, F. Guinea, and P. San-Jose, Phys. Rev. Lett. 119, 107201 (2017).

[17] A. O. Sboychakov, A. V. Rozhkov, A. L. Rakhmanov, and F. Nori, Phys. Rev. Lett. 120, 266402 (2018).

[18] A. Ramires and J. L. Lado, Phys. Rev. Lett. 121, 146801 (2018).

[19] A. Dalal and J. Ruhman, arXiv:2103.06313.

[20] Y. Cao, D. Chowdhury, D. Rodan-Legrain, O. RubiesBigorda, K. Watanabe, T. Taniguchi, T. Senthil, and P. Jarillo-Herrero, Phys. Rev. Lett. 124, 076801 (2020).

[21] S. Chen, M. He, Y.-H. Zhang, V. Hsieh, Z. Fei, K. Watanabe, T. Taniguchi, D. H. Cobden, X. Xu, C. R. Dean, and M. Yankowitz, Nat. Phys. 17, 374 (2021).

[22] J. M. Park, Y. Cao, K. Watanabe, T. Taniguchi, and P. Jarillo-Herrero, Nature (London) 590, 249 (2021).

[23] Z. Hao, A. M. Zimmerman, P. Ledwith, E. Khalaf, D. H. Najafabadi, K. Watanabe, T. Taniguchi, A. Vishwanath, and P. Kim, Science 371, 1133 (2021).

[24] J. Liu, Z. Ma, J. Gao, and X. Dai, Phys. Rev. X 9, 031021 (2019).

[25] A. Lopez-Bezanilla and J. L. Lado, Phys. Rev. Research 2, 033357 (2020).

[26] E. Khalaf, A. J. Kruchkov, G. Tarnopolsky, and A. Vishwanath, Phys. Rev. B 100, 085109 (2019).

[27] S. Carr, C. Li, Z. Zhu, E. Kaxiras, S. Sachdev, and A. Kruchkov, Nano Lett. 20, 3030 (2020).

[28] Z. Wu, Z. Zhan, and S. Yuan, Sci. China Phys. Mech. \& Astron. 64, 267811 (2021).

[29] P. Coleman, in Many-Body Physics: From Kondo to Hubbard, edited by E. Pavarini, E. Koch, and P. Coleman (Forschungszentrum Jülich $\mathrm{GmbH}$ Zentralbibliothek, Verlag Jülich, 2015), ISBN 978-3-95806-074-6.

[30] S. Wirth and F. Steglich, Nat. Rev. Mater. 1, 16051 (2016).

[31] Q. Si and F. Steglich, Science 329, 1161 (2010).

[32] A. Ramires, Nat. Phys. 15, 1212 (2019).

[33] Q. Si, Phys. Status Solidi (b) 247, 476 (2010).

[34] P. Coleman and A. H. Nevidomskyy, J. Low Temp. Phys. 161, 182 (2010).

[35] P. San-Jose and E. Prada, Phys. Rev. B 88, 121408(R) (2013)

[36] Y. Xie, B. Lian, B. Jäck, X. Liu, C.-L. Chiu, K. Watanabe, T. Taniguchi, B.A. Bernevig, and A. Yazdani, Nature (London) 572, 101 (2019).

[37] A. H. Castro Neto, F. Guinea, N. M. R. Peres, K. S. Novoselov, and A. K. Geim, Rev. Mod. Phys. 81, 109 (2009). 
[38] X. Li, F. Wu, and A. H. MacDonald, arXiv:1907.12338.

[39] E. Colomés and M. Franz, Phys. Rev. Lett. 120, 086603 (2018).

[40] A. Ramires and J. L. Lado, Phys. Rev. Lett. 121, 146801 (2018).

[41] A. Ramires and J. L. Lado, Phys. Rev. B 99, 245118 (2019).

[42] T. M. R. Wolf, J. L. Lado, G. Blatter, and O. Zilberberg, Phys. Rev. Lett. 123, 096802 (2019).

[43] T. M. R. Wolf, O. Zilberberg, G. Blatter, and J. L. Lado, Phys. Rev. Lett. 126, 056803 (2021).

[44] Y. Wang, L. G. Hector, H. Zhang, S. L. Shang, L. Q. Chen, and Z. K. Liu, Phys. Rev. B 78, 104113 (2008).

[45] A. Shick, W. Pickett, and A. Liechtenstein, J. Electron Spectrosc. Relat. Phenom. 114-116, 753 (2001).

[46] D. A. Andersson, S. I. Simak, B. Johansson, I. A. Abrikosov, and N. V. Skorodumova, Phys. Rev. B 75, 035109 (2007).

[47] B. Dorado, B. Amadon, M. Freyss, and M. Bertolus, Phys. Rev. B 79, 235125 (2009).

[48] O. Eriksson, L. Nordström, M. S. S. Brooks, and B. Johansson, Phys. Rev. Lett. 60, 2523 (1988).

[49] F. Cricchio, F. Bultmark, O. Grånäs, and L. Nordström, Phys. Rev. Lett. 103, 107202 (2009).

[50] E. Mendive-Tapia and J. B. Staunton, Phys. Rev. Lett. 118, 197202 (2017).

[51] F. Guinea and N. R. Walet, Proc. Natl. Acad. Sci. U.S.A. 115, 13174 (2018).

[52] J. M. Pizarro, M. Rösner, R. Thomale, R. Valentí, and T. O. Wehling, Phys. Rev. B 100, 161102(R) (2019).

[53] T. Cea, N. R. Walet, and F. Guinea, Phys. Rev. B 100, 205113 (2019).

[54] P. Stepanov, I. Das, X. Lu, A. Fahimniya, K. Watanabe, T. Taniguchi, F. H. L. Koppens, J. Lischner, L. Levitov, and D. K. Efetov, Nature (London) 583, 375 (2020).

[55] P. A. Pantaleon, T. Cea, R. Brown, N. R. Walet, and F. Guinea, arXiv:2003.05050.

[56] A. Liechtenstein, M. Katsnelson, V. Antropov, and V. Gubanov, J. Magn. Magn. Mater. 67, 65 (1987).

[57] A. Oswald, R. Zeller, P. J. Braspenning, and P. H. Dederichs, J. Phys. F 15, 193 (1985).

[58] S. Lounis and P. H. Dederichs, Phys. Rev. B 82, 180404(R) (2010).

[59] A. Szilva, M. Costa, A. Bergman, L. Szunyogh, L. Nordström, and O. Eriksson, Phys. Rev. Lett. 111, 127204 (2013).

[60] J. González, F. Guinea, and M. Vozmediano, Nucl. Phys. B424, 595 (1994).

[61] V. N. Kotov, B. Uchoa, V. M. Pereira, F. Guinea, and A. H. Castro Neto, Rev. Mod. Phys. 84, 1067 (2012).

[62] D. C. Elias, R. V. Gorbachev, A. S. Mayorov, S. V. Morozov, A. A. Zhukov, P. Blake, L. A. Ponomarenko, I. V. Grigorieva, K. S. Novoselov, F. Guinea, and A. K. Geim, Nat. Phys. 7, 701 (2011).

[63] E. G. Mishchenko, Phys. Rev. Lett. 98, 216801 (2007).
[64] D. E. Sheehy and J. Schmalian, Phys. Rev. Lett. 99, 226803 (2007).

[65] T. Stauber, P. Parida, M. Trushin, M. V. Ulybyshev, D. L. Boyda, and J. Schliemann, Phys. Rev. Lett. 118, 266801 (2017).

[66] J. Kang and O. Vafek, Phys. Rev. Lett. 122, 246401 (2019).

[67] K. Seo, V. N. Kotov, and B. Uchoa, Phys. Rev. Lett. 122, 246402 (2019).

[68] L. Borda, G. Zaránd, W. Hofstetter, B. I. Halperin, and J. von Delft, Phys. Rev. Lett. 90, 026602 (2003).

[69] G. Zarnd, A. Brataas, and D. Goldhaber-Gordon, Solid State Commun. 126, 463 (2003).

[70] R. López, D. Sánchez, M. Lee, M.-S. Choi, P. Simon, and K. Le Hur, Phys. Rev. B 71, 115312 (2005).

[71] M.-S. Choi, R. López, and R. Aguado, Phys. Rev. Lett. 95, 067204 (2005).

[72] M. Filippone, C. P. Moca, G. Zaránd, and C. Mora, Phys. Rev. B 90, 121406(R) (2014).

[73] E. J. König, P. Coleman, and A. M. Tsvelik, Phys. Rev. B 102, 104514 (2020).

[74] P. Coleman, Phys. Rev. B 28, 5255 (1983).

[75] A. Ramires and P. Coleman, Phys. Rev. B 93, 035120 (2016).

[76] A. Auerbach and K. Levin, Phys. Rev. Lett. 57, 877 (1986).

[77] A. J. Millis and P. A. Lee, Phys. Rev. B 35, 3394 (1987).

[78] D. P. Arovas and A. Auerbach, Phys. Rev. B 38, 316 (1988).

[79] A. K. Mitchell, D. Schuricht, M. Vojta, and L. Fritz, Phys. Rev. B 87, 075430 (2013).

[80] R. Žitko, Phys. Rev. B 81, 241414(R) (2010).

[81] M. Koshino, N. F. Q. Yuan, T. Koretsune, M. Ochi, K. Kuroki, and L. Fu, Phys. Rev. X 8, 031087 (2018).

[82] The effective exchange couplings depends on the charging energy that can be controlled via dielectric engineering.

[83] H. Zhao, J. Zhang, M. Lyu, S. Bachus, Y. Tokiwa, P. Gegenwart, S. Zhang, J. Cheng, Y.-f. Yang, G. Chen, Y. Isikawa, Q. Si, F. Steglich, and P. Sun, Nat. Phys. 15, 1261 (2019).

[84] J. Zhang, H. Zhao, M. Lv, S. Hu, Y. Isikawa, Y.-f. Yang, Q. Si, F. Steglich, and P. Sun, Phys. Rev. B 97, 235117 (2018).

[85] S. Friedemann, T. Westerkamp, M. Brando, N. Oeschler, S. Wirth, P. Gegenwart, C. Krellner, C. Geibel, and F. Steglich, Nat. Phys. 5, 465 (2009).

[86] P. Aynajian, E. H. da Silva Neto, A. Gyenis, R. E. Baumbach, J. D. Thompson, Z. Fisk, E. D. Bauer, and A. Yazdani, Nature (London) 486, 201 (2012).

[87] M. Maltseva, M. Dzero, and P. Coleman, Phys. Rev. Lett. 103, 206402 (2009).

[88] J. Figgins and D. K. Morr, Phys. Rev. Lett. 104, 187202 (2010).

[89] A. R. Schmidt, M. H. Hamidian, P. Wahl, F. Meier, A. V. Balatsky, J. D. Garrett, T. J. Williams, G. M. Luke, and J. C. Davis, Nature (London) 465, 570 (2010).

[90] S. Ernst, S. Kirchner, C. Krellner, C. Geibel, G. Zwicknagl, F. Steglich, and S. Wirth, Nature (London) 474, 362 (2011). 